\title{
What is a pediatric tumor?
}

This article was published in the following Dove Press journal:

Clinical Oncology in Adolescents and Young Adults

19 November 2012

Number of times this article has been viewed

\author{
Jaume Mora ${ }^{1,2}$ \\ 'Department of Oncology, \\ ${ }^{2}$ Developmental Tumor Biology \\ Laboratory, Hospital Sant Joan de \\ Deu, Fundacio Sant Joan de Deu, \\ Barcelona, Spain
}

Correspondence: Jaume Mora

Passeig Sant Joan de Deu, 2 ,

Esplugues de Llobregat, Barcelona,

Catalunya 08950, Spain

Tel +34 932804000

Fax +34936006II9

Email jmora@hsjdbcn.org
Abstract: Working together with medical oncologists, the question of whether a Ewing sarcoma in a 25-year-old is a pediatric tumor comes up repeatedly. Like Ewing's, some tumors present characteristically at ages that cross over what has been set as the definition of pediatrics (15 years, 18 years, or 21 years?). Pediatric oncology textbooks, surprisingly, do not address the subject of defining a pediatric tumor. They all begin with an epidemiology chapter defining the types of tumors appearing at distinct stages of childhood, adolescence, and young adulthood. Describing the epidemiology of tumors in relation to age, it becomes clear that the disease is related to the phenomenon of aging. The question, however, remains: is there a biological definition of what pediatric age is? And if so, will tumors occurring during this period of life have anything to do with such biological definition? With the aim of finding an objective definition, the fundamental concepts of what defines "pediatrics" was reviewed and then the major features of tumors arising during development were analyzed. The tumors were explored from the perspective of a host immersed in the normal process of growth and development. This physiological process, from pluripotential and undifferentiated cells, makes possible the differentiation, maturation, organization, and function of tissues, organs, and apparatus. A biological definition of pediatric tumors and the infancy-childhood-puberty classification of developmental tumors according to the infancy-childhood-puberty model of normal human development are proposed.

Keywords: growth and development, pediatric tumor, infant, childhood and adolescence, pubertal tumors

\section{Introduction}

As pediatric oncologists, it is assumed that the subject of scientific interest is well described and the type of patients cared for is clear. However, when discussing this subject over with adult medical oncologists, grey areas appear and the subject of definition becomes unclear. For example, when dealing with Ewing sarcoma (ES) tumors, where age boundaries cross over what has been set as the definition of pediatrics (15 years, 18 years, or 21 years?), one may ask what the objective definition of a pediatric tumor is - crystal clear when dealing with an infant with leukemia, but not as clear when facing a 20-year-old with ES, desmoplastic sarcoma, or non-Hodgkin's lymphoma. The first chapter of a pediatric oncology textbook seemed a good place to look for a definition. Surprisingly, a chapter dealing with such primordial definition could not be found. Notably, most pediatric oncology textbooks begin with an epidemiology chapter and define clearly the types of tumors appearing at distinct stages of childhood, adolescence, and young adulthood. Describing epidemiology in 
relation to age, it becomes clear that the disease is related to the phenomenon of aging.

In the US, pediatric oncologists typically care for patients up to 21 years of age and sometimes up to 30 years for patients with bone tumors. In Europe, pediatric practice is restricted more commonly to those under 15 or 18 years of age. Typically in the past, children were considered individuals under the age of 15 years; however, the World Health Organization classifies a child as an individual under 18 years of age. The question remains: is there a biological definition of what pediatric age is? And if so, will tumors occurring during this period of life have anything to do with such biological definition?

Ten years ago a scholarly focus on embryonic malignancies by authorities Maris and Denny proposed that "the majority of pediatric cancers likely reflect the inherent risks associated with the complex process of normal development." Does this definition apply to the 20-year-old patient with ES or any neoplasia? After all, being trained as pediatricians, it is well known that development and growth are fundamental concepts of the profession. Therefore, with the aim of finding a biological definition, general pediatrics textbooks were searched, the elementary concepts of what defines "pediatrics" were revisited, and the general features of tumors arising during infancy, childhood, and adolescence were then analyzed. The hypothesis was that if the basic concepts underlying the words "pediatric" and "cancer" could be clarified, then a conceptual definition should be able to be formed, from the current knowledge, of what a pediatric tumor is.

\section{What is pediatrics?}

Pediatrics is the branch of medicine that deals with the medical care of infants, children, and adolescents, and the fundamental characteristic of these three stages in life is growth.

\section{Growth and development}

Growth and development is a physiological process that, from a pluripotential and undifferentiated cell, makes possible the differentiation, maturation, organization, and function of tissues, organs, and apparatuses that, as a whole, make up the human body. ${ }^{2}$ As multifactorial and complex as the process of growth is, normal growth is remarkably predictable and established in three stages:

1. Cellular hyperplasia - during organogenesis and the fetal period growth is characterized by cellular division and cellular proliferation, ie, hyperplasia. It is characterized by the highest cellular replication rate in the body's biological time.

2. Hyperplasia-hypertrophy - when the organ or tissue is reaching the predetermined cellular content, a decrease in the cellular division rate occurs, a lesser growth in the number of cells, and, at the same time, an increase in the cellular size.

3. Hypertrophy - when the adult cellular content is reached, cellular division stops and cellular growth depends exclusively on the size of growth of the existing cells. After gestation week 28 growth is mainly due to hypertrophy, decreasing further the mitotic rate and progressively increasing cellular size. ${ }^{2}$

The human body initiates growth immediately after conception and continues to develop until reaching the adult height. However, each organ, tissue, and body segment grows and develops with its own sequence (at different times, with different durations, and at different rates), reaching the adult size at different chronological ages. ${ }^{2}$ For example, growth of the central nervous system is extremely rapid in the prenatal time and during the first years of postnatal life, reaching $90 \%$ of adult weight at 5 years of age. ${ }^{2}$

\section{Biologically-oriented mathematical model for human growth}

A mathematical method modeling human growth composed by three additive and partly superimposed components was proposed in 1989 by Karlberg - the infancy-childhoodpuberty (ICP) model. ${ }^{3}$ Each subcomponent reflects the different hormonal phases of the growth process. The pattern of human linear growth is very well documented and shows a sigmoid morphology (Figure 1), with a peak prenatal velocity of growth and a rapid deceleration for the first two postnatal years, followed by a period with lower and slowly decreasing velocity from the third year until puberty. The infancy component expands from the second half of pregnancy until 2-3 years of postnatal life. This period is mainly dependent on maternal nutrition, independent of hormones. The childhood component lasts from the end of infancy until the beginning of puberty. During this long period, the intense deceleration of the first 2-3 years becomes a stable slow growth rate. The beginning of this component is marked by the initial progressive influence of growth hormone $(\mathrm{GH})$ upon linear growth. The puberty component is the result of the synergy of the two hormonal systems, one dependent on $\mathrm{GH}$ and the other on sexual steroids.

Taken altogether, a precise knowledge of physiological growth, mathematically well described and predicted, has 


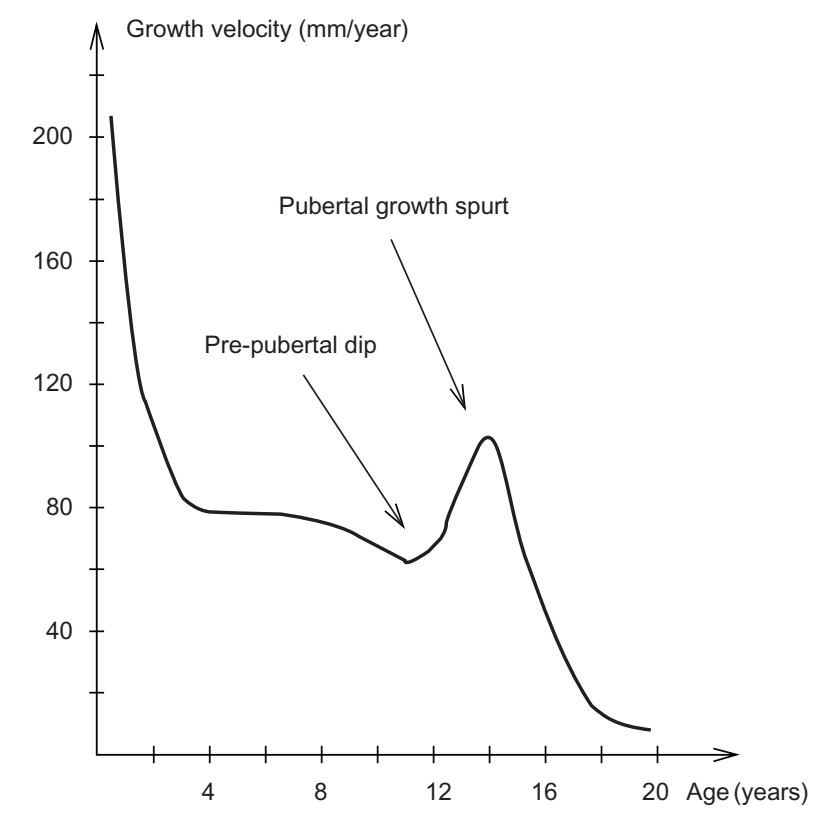

Figure I Growth velocity curve of normal human growth.

Note: A mathematical model composed of three additive and partly superimposed components (infant, childhood, and puberty; ICP model) describes the function as described by Karlberg. (C) 1989 John Wiley and Sons. Reprinted from Karlberg J.A biologically-oriented mathematical model (ICP) for human growth. Acta Paediatr Scand Suppl. ${ }^{3}$

Abbreviation: ICP, infancy-childhood-puberty.

been achieved, with three well-defined biological stages. Can these principles be used to describe the tumors that appear during development? Furthermore, can pediatric cancer be defined in terms of the forces that drive normal human growth?

\section{What is cancer?}

Many excellent definitions of cancer do exist. One that may be concise enough and comprehensive is "the territorial expansion of a mutant clone." ${ }^{\prime 4}$ Mutations, accumulated sequentially within descendent subclones over years or decades, drive the disease by natural selection of cells. Cancer development is fundamentally a dynamic Darwinian process of mutational diversification and clonal selection. However, cancer is about more than just cancer cells. Cancer can be considered an endocrine organ that affects the host at a systemic level. The features that the cancer acquires to successfully grow and metastasize to distant sites produce multiple factors that result in different clinical syndromes that may become lethal for the patient.

Cancer cells exist within a complex ecosystem populated by host cells. The many characteristics of the mutant cell and the environment in which this clone emerges define the genotype and phenotype of the eventual tumor. Cancers that develop during, and as a consequence of, the complex process of development, while body growth is active, reproduce the cells and environments from which they emerge.

\section{Phylogeny, ontogeny, and oncogenesis}

Over the past three decades it has been discovered that the genesis of cancer mirrors the process of mutation and selection underlying human evolution, and the signals that drive or suppress proliferation, apoptosis, differentiation, and quiescence in cancer cells mimic those of normal development. ${ }^{5}$ The stepwise process of generating tissues correctly in time and space is very precise and requires highly orchestrated cellular functions. It is perhaps not surprising that the error rate is substantial - most human embryos fail to implant or subsequently die. ${ }^{6}$

An embryonic theory of cancer was proposed in 1875 and is now supported by an increasing number of experimental studies. ${ }^{7-9}$ Briefly, the theory is based upon the relationship between ontogeny and oncogenesis and implies an impaired cellular maturation that would result in the overproduction of undifferentiated (stem?) cells, which then accumulate. In support of the theory is the connection between congenital anomalies and childhood cancer. Recent studies show an almost three-fold overall increased risk of malignancies like leukemia and lymphoma with congenital anomalies. ${ }^{10}$

Embryonic tumors, because they originate from immature tissue, resemble tissues in the developing embryo and fetus. Furthermore, some tumors cells not only look like embryonic cells, but they functionally mimic their behavior. For example, cells of hepatoblastoma and germ cell tumors can secrete $\alpha$-fetoprotein - a serum protein that is produced by normal fetal cells only during pregnancy. In general, the biology of embryonic cancer cells largely recapitulates the behavior of cells that are found in developing tissues. For instance, gene expression profiles of developmental tumors have been compared with those of various stages of normal tissue development. This has demonstrated the close relationship between these cancer cells and the immature cells of the developing organs from which these tumors arise: Wilms' tumors and the metanephric mesenchyma, ${ }^{11}$ neuroblastoma and sympathoadrenal progenitors of the neural crest, ${ }^{12}$ retinoblastoma and cone precursor cells of the retina, ${ }^{13}$ fetal skeletal muscle and rhabdomyosarcoma, ${ }^{14}$ hemangiomas and fetal endothelial cells,${ }^{15}$ medulloblastoma and cerebellar precursor cells, ${ }^{16}$ and gliomas and neural precursor cells. ${ }^{17}$

\section{Epidemiology meets biology}

In contrast to adult cancers, $85 \%$ of which occur in the perpetually self-renewing epithelia of various organs, cancers 
occurring during developmental ages are of a much wider spectrum, with at least $50 \%$ in the hematopoietic system and central nervous system and only $9 \%$ in epithelia. ${ }^{18}$ Acute leukemia is the principal subtype of pediatric cancer in affluent societies, where its incidence rate is within the range of $30-45$ per million children $(<15$ years) per year. In most developing or less affluent countries the rate of childhood leukemia seems to be several-fold lower. ${ }^{19}$ Despite some reports of ethnic and geographical variation, most types of pediatric tumors occur with similar worldwide prevalence. A general and accepted explanation of this observation is that they are not likely to be caused by environmental factors.

Natural selection is expected to clear out developmental errors during embryo and fetal development resulting in prereproductive deaths, but there are limits. Every round of cell proliferation involves the challenge of faithfully copying the entire genome. Proofreading DNA polymerases significantly limit errors in nucleotide incorporation. In humans, the average net mutation rate for single base changes is about $10^{-9}$ per replicative cycle and double-stranded DNA breaks occur spontaneously 50 times per cycle. ${ }^{20,21}$ The repair of double-stranded DNA breaks is intrinsically error prone and can lead inadvertently to chromosome translocations, as in leukemia and sarcoma. ${ }^{22}$ Taken together, all the spontaneous mutations occurring during the 42 division cycles of fetal development might be a sufficient explanation for embryonic cancers, without the need for any external genotoxic exposures. ${ }^{23}$ Additional risk of developmental cancer might have been acquired in the relatively recent evolutionary past by rapid changes in human growth patterns and morphology. For example, the pubertal growth spurt seems to be an evolutionary novelty and osteosarcoma in adolescents could be a byproduct of recent evolutionary changes in human growth. ${ }^{23}$

\section{ICP tumors}

Having described pediatrics and cancer in terms of development and growth, a biological definition and classification of tumors occurring during normal human development following the ICP model are proposed (Table 1).

\section{Infant tumors}

Infant tumors are defined as those arising from cellular populations that have not completed the process of terminal differentiation during fetal or postnatal development following the pattern of growth characteristic of prenatal growth, ie, hyperplasia. It is postulated that these tumors grow during the infancy component of the ICP model, ie, from the second half of pregnancy until 2-3 years of postnatal life (Figure 2). This period of growth is mainly dependent on maternal nutrition, independent of hormones, and is especially susceptible to the activity of in utero toxics. The physiological characteristics of this period define the major features of the tumors that develop, ie, rapid growth before the age of 2 years, high frequency of congenital presentations (before 3 months of age), and a high tendency to spontaneously regress. Clinically these tumors are diagnosed before 3 years of age and include tumors occurring in the neonatal period like hemangiomas, neuroblastoma, teratoma, leukemia, and brain tumors. Characteristically, they are often associated with congenital malformations. ${ }^{24,25}$ Included in this group of tumors are many of the blastomas described in all human systems (Table 1). This includes infant medulloblastoma

Table I Classification of pediatric tumors

\begin{tabular}{lll}
\hline Infant tumors & Childhood tumors & Puberty tumors \\
\hline Hemangioma & Childhood leukemia (ALL and AML) & Hodgkin's lymphoma \\
Infant neuroblastoma & Childhood astrocytoma & Gonadal germ cell tumors \\
Congenital germ cell tumors & Nephroblastoma (Wilms tumor) & Ewing sarcoma \\
Infant leukemia (ALL and AML) & Childhood neuroblastoma & Osteosarcoma \\
Infant (SHH type) medulloblastoma & Embryonal rhabdomyosarcoma & DSRCT \\
Atypical teratoid/rhabdoid tumor & Langerhans cell histiocytosis & Synovial sarcoma \\
Central PNET & Non-Hodgkin's lymphoma & Alveolar rhabdomyosarcoma \\
Ependymoblastoma & Fibromatosis & Neuroblastoma of adolescents and young adults \\
Hepatoblastoma & Group A posterior fossa ependymoma & Medulloblastoma (SHH type of young adults) \\
Retinoblastoma & & Group B posterior fossa ependymoma \\
Mesoblastic nephroma & & \\
Congenital fibrosarcoma & & \\
Inflammatory myofibroblastic tumor & & \\
Pulmonary pleuroblastoma & & \\
\hline
\end{tabular}

Abbreviations: ALL, acute lymphoblastic leukemia; AML, acute myeloblastic leukemia; DSRCT, desmoplastic small round cell tumor; PNET, primitive neuroectodermal tumor; $\mathrm{SHH}$, sonic hedgehog homolog. 


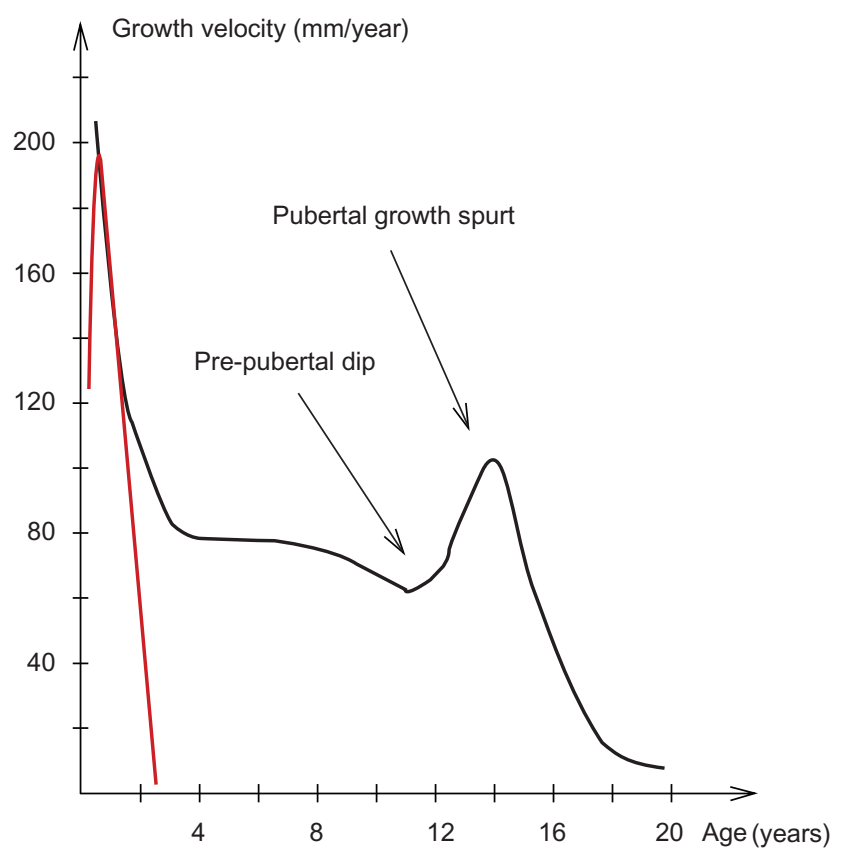

Figure 2 Overlap of the growth velocity curve of normal human growth with the age curve presentation (in red) of infant tumors.

Note: The incidence curve of infant tumors overlaps with the postnatal deceleration phase of the growth curve during the first 2 years of postnatal life.

(sonic hedgehog homolog type), atypical teratoid/rhabdoid tumor, central primitive neuroectodermal tumor, and ependymoblastoma in the central nervous system; infant neuroblastoma in the peripheral nervous system; hepatoblastoma in the gastrointestinal system; retinoblastoma in the eye; mesoblastic nephroma and the germline tumors (teratocarcinomas, yolk sac tumor, and embryonic carcinoma) in the genitourinary system; infant lymphoblastic and myeloblastic leukemia in the hematopoietic organ; congenital fibrosarcoma and inflammatory myofibroblastic tumor in the musculoskeletal system; infantile hemangioma in the vascular endothelial system; and pulmonary pleuroblastoma in the lungs.

Hemangiomas are benign tumors of the vascular endothelium and are the most common tumors of infancy, affecting $1 \%-2 \%$ of full-term infants. ${ }^{17}$ Hemangiomas can be solitary or multiple and their clinical importance is related to their location. Typically, they are not apparent at birth, but appear around the second week of life. The pattern of growth is characteristically proliferative for the first 8-12 months of life, with involution occurring over $1-5$ years and continuing to the final state at 7-10 years. Cessation of proliferation coincides with the end of the infancy period of human growth. ${ }^{17}$

Neuroblastoma in the infant has a more favorable prognosis than in older children. One reason for this is the peculiar behavior of stage $4 \mathrm{~S}$ disease (where " $\mathrm{S}$ " stands for special), which frequently undergoes spontaneous regression. In 1971, this special and rare subgroup of metastatic neuroblastoma affecting very young infants, characterized by a unique pattern of dissemination and a high incidence of spontaneous regression, was described. ${ }^{26}$ Stage $4 \mathrm{~S}$ has been recognized as a distinct clinical entity in all subsequent classifications of neuroblastoma. Like in infantile hemangiomas, stage $4 \mathrm{~S}$ neuroblastoma is a clonal expansion of mutated (specific pattern of mutations) precursor cells (neuroblasts) able to escape growth control initially and grow as large tumors until they stop proliferating at the end of the infancy period. Stage $4 \mathrm{~S}$ neuroblastoma is able to respond to differentiation signals and regress on its own. ${ }^{27}$ Like in hemangiomas, infant and congenital neuroblastomas are statistically associated with congenital anomalies and predisposing syndromes. ${ }^{24}$

Leukemias diagnosed in the first year of life are clinically and biologically distinct from those of older children. ${ }^{28}$ One major genetic distinction is the remarkably high incidence of 11q23 translocations involving the $M L L$ gene. $M L L$ rearrangements are present in $50 \%-75 \%$ of infant leukemia's versus $5 \%-10 \%$ of those occurring in older children and adults. Epidemiological and molecular genetic investigations demonstrate that $M L L$ translocations occur in utero in most cases. ${ }^{29}$ For infant acute lymphoblastic leukemia, relevant exposure and complementary genetic susceptibility factors impact exclusively in utero during fetal hematopoiesis. $M L L$ leukemias do not regress spontaneously; on the contrary, they show an aggressive behavior resulting in an overall poor outcome. ${ }^{30}$

\section{Childhood tumors}

Childhood tumors are defined as those arising from cellular populations that have not completed the process of terminal differentiation (embryonic by histopathology) and the blocking of differentiation occurs when the organ or tissue reaches the predetermined cellular content. The environment of growth is characterized by a decrease in the cellular division rate (as compared to the infancy period), lesser growth in the number of cells, and, at the same time, an increase in the cellular size, ie, characteristic features of the hyperplasiahypertrophy period. It is postulated that these tumors develop during the childhood component of the ICP model, from the end of infancy at 3 years of age until the beginning of puberty, a developing environment marked by the progressive influence of GH upon growth (Figure 3).

The most common childhood tumor is leukemia, and the most frequent subtype acute lymphoblastic leukemia shows a very marked peak of incidence at 2-5 years in the Western 


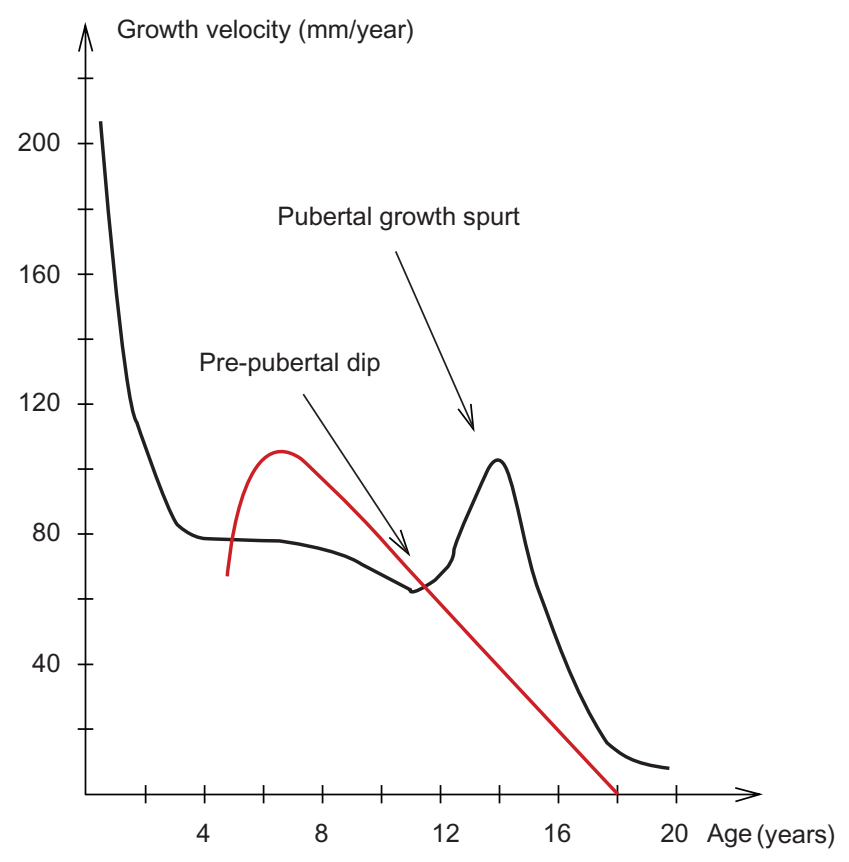

Figure 3 Overlap of the growth velocity curve of normal human growth with the age curve presentation (in red) of childhood tumors.

Note: The incidence curve of childhood tumors overlaps with the stable and slow growth phase of the growth velocity curve.

world. ${ }^{30}$ The natural history of childhood leukemia involves a two-step hit model with prenatal initiation of preleukemic clones followed by postnatal promotion, secondary mutation, and overt disease. Latency after initiation can be variable (a few months to 15 years) and animal modeling supports this two-step mechanism. ${ }^{30}$ Epidemiological evidence available is consistent with the hypothesis that childhood leukemias arise as a consequence of an abnormal immune response to common infections. ${ }^{31}$ The childhood component of human growth provides the appropriate context for childhood leukemia to occur following the development of the immune system. The thymus, peripheral lymph nodes, and Waldeyer's ring develop during the first years of postnatal life and reach $100 \%$ of adult size by 5 years of age. The growth of the immune system slowly continues over the childhood period and reaches its maximum by $12-13$ years of age $-180 \%$ of the adult size. ${ }^{2}$ During this process of differentiation and maturation, the immune system might be specially suited to tolerating abnormal proliferation of preneoplastic clones.

The second most common class of cancer during the period of childhood is astrocytoma. This high frequency may be attributed to the pattern of brain growth brain during the first years of postnatal life, reaching 90\% of adult weight at 5 years of age. Based on international estimates from the World Health Organization, low-grade glial cell malignancies account for more than $50 \%$ of all brain tumors in children up to 15 years old. ${ }^{32}$ The mean age at diagnosis ranges between 6-11 years, and the most common subtype is the World Health Organization grade I pilocytic astrocytoma, ${ }^{33}$ which comprise $85 \%$ of cerebellar gliomas and almost all gliomas within the optic nerve pathway. ${ }^{34}$ The natural history of pilocytic astrocytoma is unique as these tumors are self-containing, almost never progress to higher grade astrocytomas, and their initial growth is usually followed by a decrease in tumor activity, after which they enter a dormant phase and either remain quiescent or restart growth followed by dormancy. Typically this natural history enters a long term dormancy period by 21 years of age when human growth (and synaptogenesis) stops, except for females during pregnancy when new cycles of growth can occur. It is postulated that brain tumor formation and growth in the childhood period represents a developmental condition in which molecular changes in normal stem cells create a permissive environment for the expansion and maintenance of neoplastic cells.

Other intrinsic childhood period tumors include nephroblastoma (Wilms tumor), neuroblastoma, embryonal rhabdomyosarcoma, Langerhans cell histiocytosis, nonHodgkin's lymphomas, and fibromatosis (Table 1). Overall, they present between 3-10 years of age (Figure 3) and can be clinically distinguished from the infant group of tumors by their lower rate of proliferation, lack of spontaneous regression, and highly curable nature with conventional cytotoxic treatments.

\section{Pubertal tumors}

Puberty is characterized by the acceleration of growth rate and the maturation of sexual organs. Functionally, adulthood begins with the attainment of adult height and full reproductive maturity. When psychological features are taken into account, adulthood initiates approximately at 19 years of age in women and 21-25 years of age in men. ${ }^{35}$ This is closely related to the recent findings from neuroscientists who conclude that the human brain generates synaptic connections until the mid $20 \mathrm{~s} .{ }^{36}$

The cancer pattern in adolescents differs from children and adults, a so-called transition pattern. Nearly $90 \%$ of all invasive cancers during adolescence are Hodgkin's lymphoma, gonadal germ cell tumors, bone sarcomas, and brain tumors. This distribution contrasts with that in children and with older persons in whom epithelial malignancies (carcinomas) account for $85 \%$ of cancers. ${ }^{37}$ Cancer incidence in the 15-24-year-old group has been rising in Western populations since registries began in the 1970s, and now the total incidence is $150-200$ per million. ${ }^{38}$ 
Puberty tumors are defined as those arising from cellular populations that have not completed the process of terminal differentiation and only manifest during the environmental growth created by puberty as a result of the synergy of the GH-insulin-like growth factor (IGF) axis and sexual steroids. Table 1 describes the list of tumors that can be ascribed to the definition of puberty tumors (Figure 4).

Fully characterized tumors of puberty include the bone sarcomas (Ewing sarcoma and osteosarcoma), Hodgkin's lymphoma, and the gonadal germ cell tumors. Osteosarcoma is one of the most common cancers among adolescents and young adults. An individual has a one in 10,000 chance of developing it in the first 20 years of life, and the greatest risk occurs during the pubertal growth spurt. ${ }^{39}$ Primary tumors typically occur in the growth zones of the most rapidly growing bones in adolescents (distal femur, proximal tibia, and proximal humerus). As in dogs, osteosarcoma is related to rapid growth: $50 \%$ of osteosarcomas occur in children in the top seventy-fifth percentile for height for their age group; different bones undergo the growth spurt at slightly different ages; and the age at which each shows the greatest risk of osteosarcoma varies accordingly. ${ }^{39}$ The abrupt rise and decline in incidence of osteosarcoma during adolescence and young adulthood would be consistent with exposure occurring at a specific common time before the peak. ${ }^{40}$ Like in leukemia, in utero exposure and development seems likely for osteosarcoma. The age-incidence curve of osteosarcoma is similar to that of the vaginal clear cell ade-

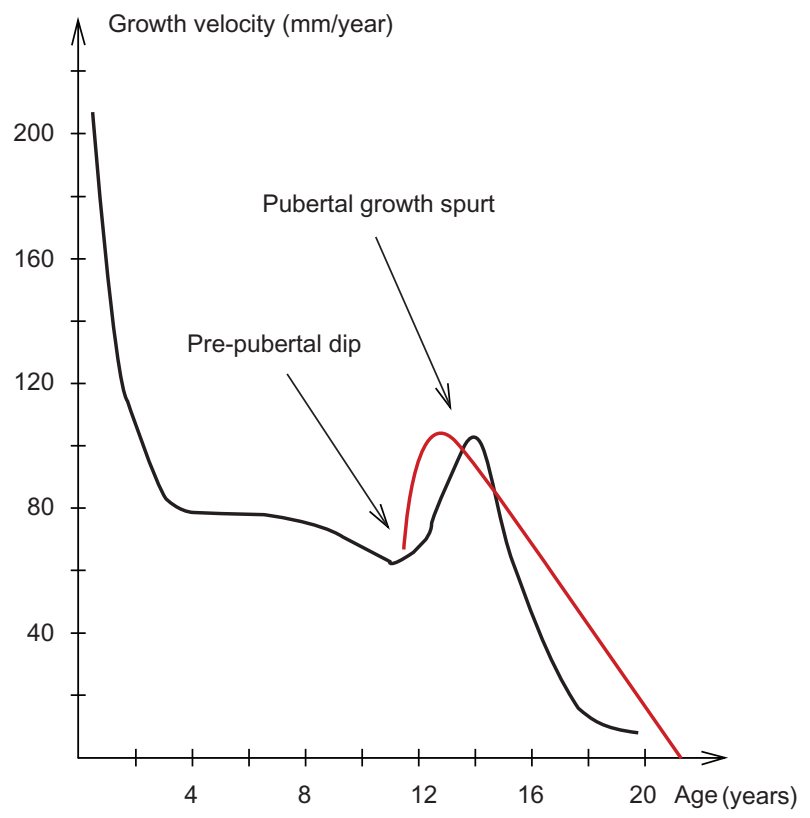

Figure 4 Overlap of the growth velocity curve of normal human growth with the age curve presentation (in red) of pubertal tumors. nocarcinoma caused by in utero diethylstilbestrol exposure. Like the adenocarcinoma, the teratogenic/carcinogenic error for osteosarcoma development occurs in the fetus, but does not manifest until the normal hormonal development and maturation of the skeleton occurs in puberty.

Patients with ES present at a median age of 14 years, with $80 \%$ younger than 18 years of age. Males are more commonly affected than females, and the disease is very rare in nonwhites. ${ }^{41}$ Ewing tumor-specific chromosomal translocations fuse EWS on chromosome 22 to a subset of ETS transcription factor family members causing two major pathways governing uncontrolled proliferation: loss of the transforming growth factor- $\beta$ inhibitory pathway and overexpression of autocrine growth stimulatory loops, mainly $\mathrm{IGF}^{41}$ Growth rate during adolescence is GH/IGF1 mediated, and evidence links IGF1 signaling with the onset of neoplasia. Examples include associations between circulating IGF1 and cancer risk and associations between growth rate in adolescence and cancer risk. ${ }^{42}$ IGF signaling plays a central role in the tumorigenesis of ES. Prieur et al showed that $E W S / F L I 1$ binds to the promoter region of IGF binding protein-3, a negative regulator of IGF1 signaling, and causes repression of its activity demonstrating a direct link between IGF1 signaling and EWS/FLII. ${ }^{43}$ Recent results of the author's group in primary ES tumor samples confirmed previous studies reporting IGF1 receptor overexpression and IGF binding protein-3 downregulation in most ES primary tumors. ${ }^{44}$ After 20 years of research clinical trials with IGF1 receptor inhibitors were initiated, which showed a wide range of activity in patients with ES with some clinically significant responses. ${ }^{45}$

\section{Conclusion}

The extraordinary growth during the embryonic-fetal period permits that from one fertilized cell at birth, $2 \times 10 \mathrm{e}^{12}$ cells are reached through 42 successive cellular divisions. During the postnatal period and until the end of growth, five more cellular divisions are added reaching a final adult cellular content of $6 \times 10 \mathrm{e}^{13}$. Experimental evidence supports that spontaneous mutations occurring during the 47 division cycles of normal human development are likely a sufficient explanation of embryonic cancers, without the need for any external genotoxic exposures. Pediatric tumors are defined as those occurring as a consequence of the complex process of physiological growth, arising from embryonic stem cells that reproduce the cells and environments from which they emerge. The ICP model of normal human development provides the adequate context for each type of embryonic 
tumor cell to grow, and allows for a biologically oriented classification of pediatric tumors.

\section{Disclosure}

The author reports no conflicts of interest in this work.

\section{References}

1. Maris JM, Denny CT. Focus on embryonal malignancies. Cancer Cell. 2002;2(6):447-450.

2. Cameron N. Human growth curve, canalization, and catch-up growth. In: Cameron N, editor. Human Growth and Development. San Diego, CA: Academic Press; 2002:1-20.

3. Karlberg J. A biologically-oriented mathematical model (ICP) for human growth. Acta Paediatr Scand Suppl. 1989;350:70-94.

4. Greaves M. Cancer causation: the Darwinian downside of past success? Lancet Oncol. 2002;3(4):244-251.

5. Scotting PJ, Walker DA, Perilongo G. Childhood solid tumours: a developmental disorder. Nat Rev Cancer. 2005;5(6):481-488.

6. Holman DJ, Wood JW. Pregnancy loss and fecundability in women. In: Ellison PT, editor. Reproductive Ecology and Human Evolution. New Brunswick, NJ: Transaction Publishers; 2011:15-38.

7. Bolande RP. Models and concepts derived from human teratogenesis and oncogenesis in early life. J Histochem Cytochem.1984;32(8): 878-884.

8. Dyer MA. Mouse models of childhood cancer of the nervous system. J Clin Pathol. 2004;57(6):561-576.

9. Gilbertson RJ. Mapping cancer origins. Cell. 2011;145(1):25-29.

10. Rankin J, Silf KA, Pearce MS, Parker L, Ward Platt M. Congenital anomaly and childhood cancer: a population-based, record linkage study. Pediatr Blood Cancer. 2008;51(5):608-612.

11. Rivera MN, Haber DA. Wilms' tumour: connecting tumorigenesis and organ development in the kidney. Nat Rev Cancer. 2005;5(9): 699-712.

12. De Preter K, Vandesompele J, Heimann P, et al. Human fetal neuroblast and neuroblastoma transcriptome analysis confirms neuroblast origin and highlights neuroblastoma candidate genes. Genome Biol. 2006;7(9):R84.

13. McEvoy J, Flores-Otero J, Zhang J, et al. Coexpression of normally incompatible developmental pathways in retinoblastoma genesis. Cancer Cell. 2011;20(2):260-275.

14. Xia SJ, Pressey JG, Barr FG. Molecular pathogenesis of rhabdomyosarcoma. Cancer Biol Ther. 2002;1(2):97-104.

15. Boye E, Yu Y, Paranya G, Mulliken JB, Olsen BR, Bischoff J. Clonality and altered behavior of endothelial cells from hemangiomas. $J$ Clin Invest. 2001;107(6):745-752.

16. Gibson P, Tong Y, Robinson G, et al. Subtypes of medulloblastoma have distinct developmental origins. Nature. 2010;468(7327):1095-1099.

17. Crouse NR, Dahiya S, Gutmann DH. Rethinking pediatric gliomas as developmental brain abnormalities. Curr Top Dev Biol. 2011;94: 283-308.

18. Gurney JG, Bondy ML. Epidemiology of childhood cancer. In: Pizzo PA, Poplack DG, editors. Principles and Practice of Pediatric Oncology. Philadelphia, PA: Lippincott Williams and Wilkins; 2006:1-13.

19. Bhatia S, Robison LL. Epidemiology of leukemia in childhood. In: Orkin SH, Fisher DE, Look AT, Lux SE, Ginsburg D, Nathan DG, editors. Oncology of Infancy and Childhood. Philadelphia, PA: Saunders Elsevier; 2009:3-25.

20. Khanna KK, Jackson SP. DNA double-strand breaks: signaling, repair and the cancer connection. Nat Genet. 2001;27(3):247-254.

21. Vilenchik MM, Knudson AG. Endogenous DNA double-strand breaks: production, fidelity of repair, and induction of cancer. Proc Natl Acad Sci U S A. 2003;100(22):12871-12876.

22. Greaves MF, Wiemels J. Origins of chromosome translocations in childhood leukaemia. Nat Rev Cancer. 2003;3(9):639-649.
23. Leroi AM, Koufopanou V, Burt A. Cancer selection. Nat Rev Cancer. 2003;3(3):226-231.

24. Munzer C, Menegaux F, Lacour B, et al. Birth-related characteristics, congenital malformation, maternal reproductive history and neuroblastoma: the ESCALE study (SFCE). Int $J$ Cancer. 2008;122(10): 2315-2321.

25. Carozza SE, Langlois PH, Miller EA, Canfield M. Are children with birth defects at higher risk of childhood cancers? Am J Epidemiol. 2012;175(12):1217-1224

26. D'Angio GJ, Evans AE, Koop CE. Special pattern of widespread neuroblastoma with a favourable prognosis. Lancet. 1971;1(7708): $1046-1049$

27. Lavarino C, Cheung NK, Garcia I, et al. Specific gene expression profiles and chromosomal abnormalities are associated with infant disseminated neuroblastoma. BMC Cancer. 2009;9:44.

28. Greaves MF. Infant leukaemia biology, aetiology and treatment. Leukemia. 1996;10(2):372-377.

29. Ford AM, Ridge SA, Cabrera ME, et al. In utero rearrangements in the trithorax-related oncogene in infant leukaemias. Nature. 1993; 363(6427):358-360.

30. Greaves MF. Aetiology of acute leukemia. Lancet. 1997;349(9048): 344-349.

31. Greaves MF, Alexander FE. An infectious etiology for common acute lymphoblastic leukemia in childhood? Leukemia. 1993;7(3): 349-360.

32. Louis DN, Ohgaki H, Wiestler OD, et al. The 2007 WHO classification of tumours of the central nervous system. Acta Neuropathol. 2007;114(2):97-109.

33. Sievert AJ, Fisher MJ. Pediatric low-grade gliomas. J Child Neurol. 2009;24(11):1397-1408.

34. Freeman CR, Farmer JP, Montes J. Low-grade astrocytomas in children: evolving management strategies. Int J Radiat Oncol Biol Phys. 1998;41(5):979-987.

35. Albritton K, Bleyer WA. The management of cancer in the older adolescent. Eur J Cancer. 2003;39(18):2584-2599.

36. Gogtay N, Giedd JN, Lusk L, et al. Dynamic mapping of human cortical development during childhood through early adulthood. Proc Natl Acad Sci U S A. 2004;101(21):8174-8179.

37. Bleyer A, Barr R, Hayes-Lattin B, Thomas D, Ellis C, Anderson B. The distinctive biology of cancer in adolescents and young adults. Nat Rev Cancer. 2008;8(4):288-298.

38. Birch JM, Alston RD, Kelsey AM, Quinn MJ, Babb P, McNally RJ. Classification and incidence of cancers in adolescents and young adults in England 1979-1997. Br J Cancer. 2002;87(11): $1267-1274$

39. Janeway KA, Gorlick R, Bernstein ML. Osteosarcoma. In: Orkin SH, Fisher DE, Look AT, Lux SE, Ginsburg D, Nathan DG, editors. Oncology of Infancy and Childhood. Philadelphia, PA: Saunders Elsevier; 2009:871-910.

40. Troisi R, Masters MN, Joshipura K, Douglass C, Cole BF, Hoover RN. Perinatal factors, growth and development, and osteosarcoma risk. $\mathrm{Br}$ J Cancer. 2006;95(11):1603-1607.

41. Rodriguez-Galindo C, Navid F, Khoury J, Krasin M. Ewing sarcoma family of tumors. In: Pappo A, editor. Pediatric Bone and Soft Tissue Sarcomas. Berlin: Springer-Verlag; 2006:181-218.

42. Gualberto A, Pollak M. Emerging role of insulin-like growth factor receptor inhibitors in oncology: early clinical trial results and future directions. Oncogene. 2009;28(34):3009-3021.

43. Prieur A, Tirode F, Cohen P, Delattre O. EWS/FLI-1 silencing and gene profiling of Ewing cells reveal downstream oncogenic pathways and a crucial role for repression of insulin-like growth factor binding protein 3. Mol Cell Biol. 2004;24(16):7275-7283.

44. Mora J, Rodriguez E, de Torres C, et al. Activated growth signaling pathway expression in Ewing sarcoma and clinical outcome. Pediatr Blood Cancer. 2012;58(4):532-538.

45. Toretsky JA, Gorlick R. IGF-1R targeted treatment of sarcoma. Lancet Oncol. 2010;11(2):105-106. 
Clinical Oncology in Adolescents and Young Adults

Dovepress

\section{Publish your work in this journal}

Clinical Oncology in Adolescents and Young Adults is an international, peer-reviewed, open access journal publishing original research, reports, editorials, reviews and commentaries on all aspects of epidemiology, diagnosis and treatment of cancers in adolescents and young adults. The manuscript management system is completely online and includes a very quick and fair peer-review system. Visit http://www.dovepress.com/testimonials.php to read real quotes from published authors.

\footnotetext{
Submit your manuscript here: http://www.dovepress.com/clinical-oncology-in-adolescents-and-young-adults-journal
} 